\title{
Intraventricular Tigecycline as a Last Resort Therapy in a Patient with Difficult-to-Treat Healthcare-Associated Acinetobacter baumannii Ventriculitis: a Case Report
}

\author{
Mohammad Abdallah ${ }^{1}$ (D) Hamzeh Alsaleh ${ }^{2} \cdot$ Abdallah Baradwan $^{3} \cdot$ Reem Alfawares $^{4} \cdot$ Abdulaziz Alobaid $^{1}$. \\ Akram Rasheed $^{5}$ - Ibrahim Soliman ${ }^{6}$
}

Accepted: 21 July 2020 / Published online: 9 August 2020

(C) Springer Nature Switzerland AG 2020

\begin{abstract}
Healthcare-associated ventriculitis and meningitis is a common complication in patients who suffer from head trauma or have undergone a neurosurgery. Healthcare-associated ventriculitis and meningitis is associated with significant morbidity and mortality. Complications of healthcare-associated ventriculitis and meningitis include persistent vegetative state, moderate and severe disability, and death. Acinetobacter baumannii is the causative pathogen in 3.6-11.2\% of cases of healthcare-associated ventriculitis and meningitis. Cases of difficult-to-treat healthcare-associated A. baumannii ventriculitis and meningitis are being reported more frequently. However, in most of these cases, a combination of intravenous (IV) and intraventricular (IVT)/intrathecal colistin achieves good therapeutic outcome. This report describes a clinical case of difficult-to-treat healthcare-associated A. baumannii ventriculitis. The A. baumannii strain was sensitive to colistin and trimethoprim-sulfamethoxazole, intermediate to tigecycline, and resistant to other antibiotics. While colistin was the drug of choice in our case, the patient developed anaphylactoid reaction during the IV administration of the loading dose of colistin, which mandated us to discontinue colistin and complicated the treatment of our patient. The patient did not respond to a combination of IV antibiotics that included meropenem, trimethoprim-sulfamethoxazole, and tigecycline. However, when IVT tigecycline was added as a last-resort therapeutic option, the patient's ventriculitis dramatically improved, and the patient was discharged from the hospital. Physicians who treat patients with healthcare-associated A. baumannii ventriculitis might resort to IVT tigecycline when they run out of therapeutic options.
\end{abstract}

Keywords Tigecycline $\cdot$ Intraventricular $\cdot$ Ventriculitis $\cdot$ Acinetobacter baumannii $\cdot$ Anaphylactoid reaction

This article is part of the Topical Collection on Medicine

Mohammad Abdallah

mohasulmoha@yahoo.com

1 Pharmaceutical Care Services, King Saud Medical City, Riyadh, Saudi Arabia

2 Pharmacy Department, Hamad Medical Corporation, Doha, Qatar

3 The Division of Infectious Diseases, King Saud Medical City, Riyadh, Saudi Arabia

4 Doha, Qatar

5 Department of Nursing Education and Development, King Saud Medical City, Riyadh, Saudi Arabia

6 Critical Care Department, King Saud Medical City, Riyadh, Saudi Arabia

\section{Introduction}

Healthcare-associated ventriculitis and meningitis is a common complication in patients who suffer from head trauma or have undergone a neurosurgery. Healthcare-associated ventriculitis and meningitis is associated with significant morbidity and mortality. Complications include persistent vegetative state, moderate and severe disability, and death [1]. Acinetobacter baumannii is the causative pathogen in 3.6$11.2 \%$ of cases of healthcare-associated meningitis [2]. The latest Infectious Diseases Society of America guidelines for the management of healthcare-associated ventriculitis and meningitis recommend meropenem for treatment of cases infected with Acinetobacter species, while polymyxins are recommended for carbapenem-resistant strains [3]. Also, the guidelines recommend the addition of intraventricular (IVT) or intrathecal administration of polymyxins for patients with healthcare-associated A. baumannii meningitis and 
ventriculitis that are difficult- to -treat with intravenous (IV) polymyxins [3].

Cases of difficult-to-treat healthcare-associated A. baumannii ventriculitis and meningitis are being reported more frequently [4]. However, in most of these cases, a combination of IV and IVT/intrathecal colistin achieves good therapeutic outcome [5, 6]. Treatment of healthcare-associated A. baumannii ventriculitis and meningitis can be complicated by the development of antimicrobial resistance (especially for meropenem and colistin) [7, 8], the development of side effects such as neurotoxicity secondary to colistin [7], or by failure of antibiotic therapy despite in vitro sensitivity (probably because of insufficient penetration of antibiotics into ventricles and meninges) [9]. This report describes a clinical case of difficult-to-treat healthcare-associated A. baumannii ventriculitis where IV and IVT colistin was not an appropriate option because of the occurrence of anaphylactoid reaction, and the patient did not respond to a combination of IV antibiotics that included meropenem, trimethoprim-sulfamethoxazole, and tigecycline. However, the infection was successfully eradicated following the addition of IVT tigecycline.

\section{Case Description}

A 56-year old man with history of diabetes mellitus and uncontrolled hypertension was presented to the emergency department with vomiting and headache. His Glasgow coma scale (GCS) was 13/15. Computed tomography (CT) scan of the brain was done; the patient had intracerebral hemorrhage (ICH) in the right basal ganglia, intraventricular hemorrhage (IVH), and hydrocephalus (Fig. 1). The patient was shifted to the operation room for external ventricular drain (EVD) placement. After operation, the patient was shifted to the intensive care unit (ICU) for monitoring on face mask (6 1 per min). On the second day, the patient had breathing difficulty and desaturated. Chest x-ray was done and showed pulmonary edema. Also, echocardiogram was done and showed diastolic dysfunction. So, the patient was intubated and connected to mechanical ventilator. After 4 days of admission, GCS improved to $15 / 15$, and the patient was extubated. On day 6 , CT brain was repeated; there was a decrease in the size of IVH.

On day 8 , the patient became highly febrile (temperature was $39.6{ }^{\circ} \mathrm{C}$ ). A cerebrospinal fluid (CSF) culture was sent, EVD was removed, and the patient was started empirically on meropenem 2 g IV q8 hours plus vancomycin 1 g IV q12 hours. Two days later, the CSF culture report showed the growth of multidrug-resistant (MDR) A. baumannii that was only sensitive to colistin and trimethoprim-sulfamethoxazole, while intermediate to tigecycline (Table 1). A. baumannii was identified by using the VITEK 2 system (bioMérieux, Marcy l'Étoile, France). Susceptibility to antibiotics was verified by disc diffusion method, and it was interpreted according to the Clinical and Laboratory Standards Institute criteria [10]. The patient was prescribed IV colistin 9 million units stat followed by 3 million units IV q8 hours. The patient developed anaphylactoid reaction during the administration of the stat dose of colistin; he had hypotension, tachycardia, restlessness, shivering, and wheezing. Colistin was discontinued, and the patient was given adrenaline $(0.5-\mathrm{mg}$ IV stat followed by $0.5-\mathrm{mcg} / \mathrm{kg} /$ min IV infusion), hydrocortisone (200-mg IV stat followed by 50-mg IV q6 hours), and diphenhydramine (50-mg IV stat followed by cetirizine $10 \mathrm{mg}$ by nasogastric tube q24 hours). The infectious disease team was consulted; the team recommended to stop vancomycin and to provide high-dose tigecycline (200-mg IV stat dose followed by 100-mg IV q12 hours). On day 13, the patient's level of consciousness decreased (GCS decreased from $15 / 15$ to $6 / 15$ ). The patient was reintubated and trimethoprim-sulfamethoxazole (1920-mg IV q6 hours) was added to the patient's antibiotic regimen. CT brain was done and showed decrease in the fresh blood inside the left lateral ventricle and mild decrease in the hemorrhagic parenchymal blood in the right basal ganglia (Fig. 1). CSF samples were withdrawn through lumbar puncture and sent for analysis. The patient had neutrophilic pleocytosis; WBC count was 4419 , and polymorphic cells percentage was $98.1 \%$. The CSF sample for chemistry analysis was hemolyzed.

On day 14, CSF culture was sent. The report came after 3 days; it showed the growth of MDR A. baumannii with antimicrobial susceptibility pattern that was identical to the first report of MDR A. baumannii in CSF (sent on day 8). The patient did not improve on the prescribed antibiotics (meropenem, trimethoprim-sulfamethoxazole, and high-dose tigecycline); fever was persistent; CSF analysis was sent, and results were abnormal (WBC count was 485 [91.7\% was polymorphic cells], glucose was low $[0.9 \mathrm{mmol} / \mathrm{L}]$, and protein was high [182.2 mg/dL]); the level of consciousness did not improve; and the patient had leukocytosis. The infectious disease team was consulted; the team recommended to extend meropenem infusion rate to 3 hours instead of $30 \mathrm{~min}$, to insert EVD, and to administer IVT tigecycline. We called the patient's family to get their permission before we administer IVT tigecycline. On day 18, EVD was inserted, and the patient received IVT tigecycline as a last therapeutic option to treat his A. baumannii ventriculitis. Written informed consent was obtained from the patient's family before administering IVT tigecycline. IVT tigecycline was prescribed as $2 \mathrm{mg}$ q12 hours. The CSF drain was closed temporarily for 4 hours after each dose of IVT tigecycline. After 8 hours of administering the first dose of IVT tigecycline, the patient developed myoclonic seizures for $4 \mathrm{~min}$. The patient received $5 \mathrm{mg}$ of IV midazolam and loading dose of IV phenytoin $(1.5 \mathrm{~g})$. CT brain was performed; compared with the previous $\mathrm{CT}$, there was mild reduction in the amount of the IVH, and no other changes were noticed (Fig. 1). The neurology team was consulted; the team ordered electroencephalogram (EEG) and started the 
Fig. 1 Computed tomography (CT) scan of the brain. A On day 1. B On day 13. C On day 19. D On day 53 a) On day one

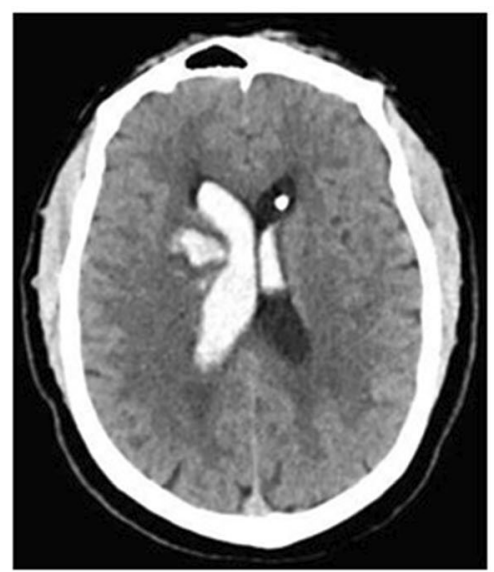

c) On day 19

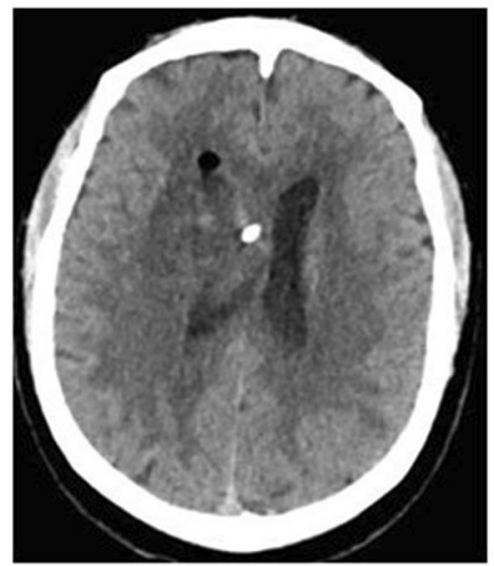

b) On day 13

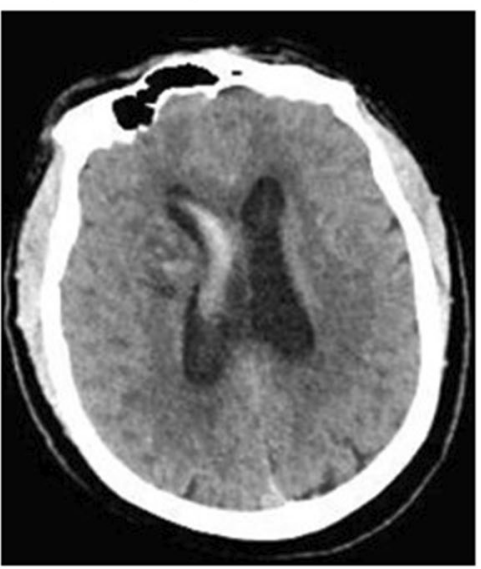

d) On day 53

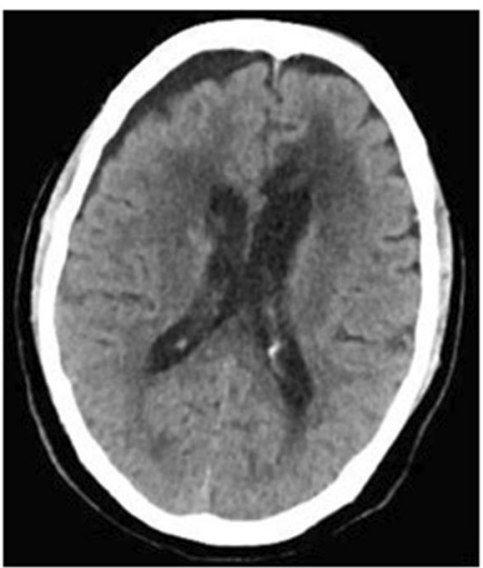

patient on levetiracetam $(500 \mathrm{mg}$ by nasogastric tube q12 hours) plus maintenance dose of phenytoin (100 mg IV q8 hours). The EEG report was abnormal and consistent with mild to moderate encephalopathy. The report mentioned that abnormal EEG could be related to metabolic, toxic, or underlying dysfunctional etiology. Moreover, no epileptiform discharges were seen in the EEG. The infectious disease team recommended to continue current regimen (meropenem, trimethoprim-sulfamethoxazole, high dose tigecycline, and IVT tigecycline) and to repeat CSF culture after 3 days. On day 21, IVT tigecycline dose was increased to $4 \mathrm{mg}$ q 12 hours because of persistent fever, leukocytosis, and low GCS. CSF culture was sent and CSF analysis was performed on day 23; the culture report showed no bacterial growth while the CSF analysis showed a decrease in protein to $75.4 \mathrm{mg} / \mathrm{dL}$, an increase in glucose to $4.3 \mathrm{mmol} / \mathrm{L}$, and a slight increase in WBC count to 536. Later on, GCS improved, temperature became normal, white blood cell count decreased, and the patient was extubated. After 37 days of ICU admission, the patient was transferred to the ward fully conscious then discharged from the hospital. All antibiotics were discontinued on day 32 of admission. The duration of antibiotics was 24 days for meropenem, 22 days for IV tigecycline, 19 days for trimethoprim-sulfamethoxazole, and 14 days for IVT tigecycline.

\section{Discussion}

Tigecycline (a glycylcycline antibiotic) has an excellent activity against several species of MDR Gram-negative and Grampositive bacteria [11]. Tigecycline was approved in 2005 by the FDA for the treatment of community-acquired pneumonia, complicated skin and skin structure infections, and complicated intra-abdominal infections. Because of its excellent effectiveness against MDR Gram-negative and Gram-positive bacteria, its safety profile, its preservative-free formulation, its synergistic effect with many antibiotics, and limited penetration to the central nervous system, IVT tigecycline is considered an attractive and promising last-resort therapeutic option for treating patients with healthcare-associated bacterial ventriculitis and meningitis [4].

Our patient was diagnosed with healthcare-associated ventriculitis after EVD insertion. The CSF culture reports (sent on day 8 and 14) showed the growth of MDR 
Table 1 Antimicrobial susceptibility pattern of the two MDR Acinetobacter baumannii isolates (isolated on day 8 and day 14 of admission)

\begin{tabular}{lll}
\hline Antimicrobial & MIC $(\mathrm{mg} / \mathrm{L})$ & Interpretation \\
\hline Amikacin & $>32$ & $\mathrm{R}$ \\
Amoxicillin-clavulanate & $>16 / 8$ & $\mathrm{R}$ \\
Ampicillin & $>16$ & $\mathrm{R}$ \\
Aztreonam & $>16$ & $\mathrm{R}$ \\
Cefepime & $>16$ & $\mathrm{R}$ \\
Ceftazidime & $>16$ & $\mathrm{R}$ \\
Ceftriaxone & $>32$ & $\mathrm{R}$ \\
Cefuroxime & $>16$ & $\mathrm{R}$ \\
Cephalothin & $>16$ & $\mathrm{R}$ \\
Ciprofloxacin & $>2$ & $\mathrm{R}$ \\
Colistin & $\leq 1$ & $\mathrm{~S}$ \\
Ertapenem & $>4$ & $\mathrm{R}$ \\
Gentamicin & $>8$ & $\mathrm{R}$ \\
Imipenem & $>8$ & $\mathrm{R}$ \\
Levofloxacin & $>4$ & $\mathrm{R}$ \\
Meropenem & $>8$ & $\mathrm{R}$ \\
Nitrofurantoin & $>64$ & $\mathrm{R}$ \\
Piperacillin-tazobactam & $>64 / 4$ & $\mathrm{R}$ \\
Tigecycline & 4 & $\mathrm{I}$ \\
Trimethoprim-sulfamethoxazole & $2 / 32$ & $\mathrm{~S}$ \\
\hline & & \\
\hline
\end{tabular}

$M I C$ minimum inhibitory concentration, $M D R$ multidrug resistant

A. baumannii that was only sensitive to colistin and trimethoprim-sulfamethoxazole, while it was intermediate to tigecycline and resistant to other antibiotics. We did not give IVT colistin because the preservative free form of colistin was not available in our hospital. The patient developed anaphylactoid reaction during the loading dose of IV colistin, which forced us to stop it and to use alternative antibiotics. Anaphylactoid reaction after colistin administration is rare but it is documented in the literature [12]. The use of trimethoprim-sulfamethoxazole is currently not recommended unless where there is no other better alternative [13]. We used trimethoprim-sulfamethoxazole $20 \mathrm{mg}$ per $\mathrm{kg}$ since the CSF culture reports showed MDR A. baumannii that was sensitive to it, and we ran out of options, especially after the anaphylactoid reaction caused by colistin. IVT tigecycline was recently used by some clinicians as a last resort to treat healthcare-associated A. baumannii ventriculitis and meningitis [4]. Our patient did not respond to a combination therapy that included meropenem, trimethoprim-sulfamethoxazole, and high-dose tigecycline. We administered IVT tigecycline $2 \mathrm{mg}$ q 12 hours for 3 days then we increased the dose to $4 \mathrm{mg}$ q12 hours. The addition of IVT tigecycline was successful in eradicating the MDR A. baumannii in the CSF. Also, signs of infection disappeared. However, our patient developed myoclonic seizures after 8 hours of administering the first dose of IVT tigecycline. Levetiracetam and phenytoin was prescribed to terminate the myoclonic seizures. Late seizures are defined as seizures occurring 1 week or more after ICH. The incidence of late seizures after ICH is almost 4 new cases/ 100 person-years, while the median delay between ICH and late seizures is 9 months (interquartile range is 3-23 months) [14]. The sole independent factor associated with the occurrence of late seizures after ICH is cortical involvement of the ICH [15]. Because the patient developed myoclonic seizures after 18 days of $\mathrm{ICH}$, and because of the absence of cortical involvement of the ICH, ICH was excluded as a precipitating factor of the myoclonic seizures. The rate of seizures associated with meropenem is less than $1 \%$ [16]. The patient developed myoclonic seizures 10 days after initiating meropenem, which excludes it a as a precipitating factor of the myoclonic seizures. However, the use of beta-lactam antibiotics (e.g., meropenem) decreases the seizure threshold [17]. The uncontrolled ventriculitis is the most likely precipitating factor of myoclonic seizures in our patient.

\section{Conclusion}

Difficult-to-treat ventriculitis caused by A. baumannii is of increasing concern due to the emergent resistance of A. baumannii to carbapenems and polymyxins, occurrence of side effects, and the insufficient penetration of many antibiotics into the ventricles. Our patient developed anaphylactoid reaction following the stat dose of IV colistin, and did not respond to a combination of IV antibiotics that included meropenem, trimethoprim-sulfamethoxazole, and tigecycline. However, when IVT tigecycline was added as a last resort, the patient's ventriculitis dramatically improved, and the patient was discharged from the hospital. Physicians who treat patients with healthcare-associated A. baumannii ventriculitis might resort to IVT tigecycline when they run out of therapeutic options.

Acknowledgments We thank the microbiology department for providing the necessary data to write this case report.

\section{Compliance with Ethical Standards}

Conflict of Interest The authors declare that there is no conflict of interest.

Ethical Approval The report was approved by the institutional review board at King Saud Medical City (H2RI-07-Oct19-01).

Informed Consent Written informed consent was obtained from the patient's family before administering IVT tigecycline and for the publication of the case report. 


\section{References}

1. Srihawan C, Castelblanco RL, Salazar L, Wootton SH, Aguilera E, Ostrosky-Zeichner L, et al. Clinical characteristics and predictors of adverse outcome in adult and pediatric patients with healthcareassociated ventriculitis and meningitis. Open Forum Infect Dis. 2016;3(2):ofw077.

2. Karaiskos I, Galani L, Baziaka F, Giamarellou H. Intraventricular and intrathecal colistin as the last therapeutic resort for the treatment of multidrug-resistant and extensively drug-resistant Acinetobacter baumannii ventriculitis and meningitis: a literature review. Int J Antimicrob Agents. 2013;41(6):499-508.

3. Tunkel AR, Hasbun R, Bhimraj A, Byers K, Kaplan SL, Scheld WM, et al. 2017 Infectious Diseases Society of America's clinical practice guidelines for healthcare-associated ventriculitis and meningitis. Clin Infect Dis. 2017;64(6):e34-65.

4. Abdallah $\mathrm{M}$, Alsaleh $\mathrm{H}$. A review of safety and effectiveness of intravenous and Intraventricular tigecycline in healthcareassociated Acinetobacter baumannii meningitis and ventriculitis. Curr Treat Options Infect Dis. 2019;11(4):331-43.

5. Fotakopoulos G, Makris D, Chatzi M, Tsimitrea E, Zakynthinos E, Fountas K. Outcomes in meningitis/ventriculitis treated with intravenous or intraventricular plus intravenous colistin. Acta Neurochir. 2016;158(3):603-10.

6. Chusri S, Sakarunchai I, Kositpantawong N, Panthuwong S, Santimaleeworagun W, Pattharachayakul S, et al. Outcomes of adjunctive therapy with intrathecal or intraventricular administration of colistin for post-neurosurgical meningitis and ventriculitis due to carbapenem-resistant acinetobacter baumannii. Int J Antimicrob Agents. 2018;51(4):646-50.

7. Lauretti L, D'Alessandris QG, Fantoni M, D'Inzeo T, Fernandez E, Pallini R, et al. First reported case of intraventricular tigecycline for meningitis from extremely drug-resistant Acinetobacter baumannii. J Neurosurg. 2017;127(2):370-3.
8. Liu Y, Pu Z, Zhao M. Case report of successful treatment of extensively drug-resistant Acinetobacter baumannii ventriculitis with intravenous plus intraventricular tigecycline. Antimicrob Agents Chemother. 2018;62(11):e01625-18.

9. Tsolaki V, Karvouniaris M, Manoulakas E, Kotlia P, Karadontas V, Fotakopoulos G, et al. Intraventricular CNS treatment with colistintigecycline combination: a case series. J Crit Care. 2018;47:338-41.

10. CLSI. Performance Standards for Antimicrobial Susceptibility Testing. 28th. CLSI supplement M100. Wayne: Clinical and Laboratory Standards Institute; 2018.

11. Pankey GA. Tigecycline. J Antimicrob Chemother. 2005;56(3): 470-80.

12. Zhan Y, Ma N, Liu R, Wang N, Zhang T, He L. Polymyxin B and polymyxin $\mathrm{E}$ induce anaphylactoid response through mediation of Mas-related G protein-coupled receptor X2. Chem Biol Interact. 2019;308:304-11.

13. Falagas ME, Vardakas KZ, Roussos NS. Trimethoprim/ sulfamethoxazole for Acinetobacter spp.: a review of current microbiological and clinical evidence. Int $\mathrm{J}$ Antimicrob Agents. 2015;46(3):231-41.

14. Rossi C, De Herdt V, Dequatre-Ponchelle N, Hénon H, Leys D, Cordonnier C. Incidence and predictors of late seizures in intracerebral hemorrhages. Stroke. 2013;44(6):1723-5.

15. Moulin S, Cordonnier C. Prognosis and outcome of intracerebral haemorrhage. Front Neurol Neurosci. 2015;37:182-92.

16. Miller AD, Ball AM, Bookstaver PB, Dornblaser EK, Bennett CL. Epileptogenic potential of carbapenem agents: mechanism of action, seizure rates, and clinical considerations. Pharmacotherapy. 2011;31(4):408-23.

17. Schliamser SE, Cars O, Norrby SR. Neurotoxicity of $\beta$-lactam antibiotics: predisposing factors and pathogenesis. J Antimicrob Chemother. 1991;27(4):405-25.

Publisher's Note Springer Nature remains neutral with regard to jurisdictional claims in published maps and institutional affiliations. 